\title{
The effect of timing on community acquired respiratory virus infection mortality during the first year after allogeneic hematopoietic stem cell transplantation: a prospective epidemiological survey
}

\author{
José Luis Piñana ${ }^{1,2} \cdot$ Ariadna Pérez $^{3} \cdot$ Juan Montoro $^{1} \cdot$ Rafael Hernani $^{3} \cdot$ Ignacio Lorenzo $^{1} \cdot$ Estela Giménez $^{4}$. \\ María Dolores Gómez ${ }^{5}$. Manuel Guerreiro ${ }^{1}$ - Eva María González-Barberá ${ }^{5}$. Carlos Carretero ${ }^{1}$. Miguel Salavert ${ }^{6}$. \\ Aitana Balaguer-Roselló ${ }^{1} \cdot$ Guillermo Sanz $\mathbb{D}^{1,2} \cdot$ Juan Carlos Hernández-Boluda $\mathbb{D D}^{3,7} \cdot$ Carlos Solano (D) $^{3,7}$. \\ Jaime Sanz ${ }^{1,2} \cdot$ David Navarro (iD ${ }^{4,8}$
}

Received: 31 May 2019 / Revised: 10 July 2019 / Accepted: 27 July 2019 / Published online: 24 September 2019

(c) The Author(s), under exclusive licence to Springer Nature Limited 2019

\begin{abstract}
The effect of timing of community acquired respiratory virus (CARV) infection after allogeneic hematopoietic stem cell transplant (allo-HCT) is an as yet unsettled issue. We evaluate this issue by including all consecutive allo-HCT recipients with molecularly-documented CARV infection during the first year after transplant. The study cohort was drawn from a prospective longitudinal survey of CARV in allo-HCT recipient having respiratory symptoms conducted from December 2013 to December 2018 at two Spanish transplant centers. Respiratory viruses in upper and/or lower respiratory specimens were tested using multiplex PCR panel assays. The study cohort comprised 233 allo-HCT recipients with $376 \mathrm{CARV}$ infection episodes diagnosed during the first year after allo-HCT. Overall, $60 \%$ of CARV episodes occurred within the first 6 months (227 out of 376). Thirty patients (13\%) had died at 3 months after CARV detection, of which $25(83 \%)$ were recipients developing CARV within the first 6 months after transplant. Multivariate analysis identified four risk factors for mortality: ATG used as part of conditioning regimen [odds ratio (OR) $2.8,95 \%$ confidence interval (C.I.) 1.21-6.4, $p=0.01$ ], CARV lower respiratory tract disease (OR 3.4, 95\% C.I. 1.4-8.4, $p=$ 0.007), CARV infection within the first 6 months of transplant (OR 3.04, 95\% C.I. 1.1-8.7, $p=0.03$ ), and absolute lymphocyte count $<0.2 \times 109 / \mathrm{L}$ (OR 2.4, 95\% C.I. $1-5.3, p=0.04$ ). Developing CARV infection within the first 6 months was associated with higher mortality. Our data supports that the timing of CARV development after allo-HCT could be of major interest.
\end{abstract}

These authors contributed equally: Jaime Sanz and David Navarro

José Luis Piñana

jlpinana@gmail.com

1 Hematology Department, Hospital Universitari i Politècnic La Fe, Valencia, Spain

2 CIBERONC, Instituto Carlos III, Madrid, Spain

3 Hematology Department, Hospital Clínico Universitario, INCLIVA Research Institute, Valencia, Spain

4 Microbiology Department, Hospital Clínico Universitario, INCLIVA Research Institute, Valencia, Spain

\section{Introduction}

Understanding the epidemiology and timing of respiratory virus infections (RVI) after allogeneic hematopoietic stem cell transplantation (allo-HCT) is important for

5 Microbiology Department, Hospital Universitari i Politècnic La Fe, Valencia, Spain

6 Department of Infectious Diseases, Hospital Universitari i Politècnic La Fe, Valencia, Spain

7 Department of Medicine, School of Medicine, University of Valencia, Valencia, Spain

8 Department of Microbiology, School of Medicine, University of Valencia, Valencia, Spain 
appropriate preventive strategies, effective diagnosis, accurate risk assessment of severe disease course, and/or treatment decision making in individual patients. The epidemiology of community acquired respiratory virus (CARV) infections among allo-HCT closely parallels incidence of infection in the community [1], although in immunocompromised patients these infections are notable for prolonged viral shedding, higher rates of pneumonia, late airflow obstruction, and mortality [2,3].

Established and validated risk factors for progression from upper respiratory tract disease (URTD) to lower respiratory tract disease (LRTD) and mortality include recipient age, smoking history, high APACHE II score, lymphopenia, high-dose total body irradiation, high-dose steroids, and presence of copathogens [4-8]. Validated risk scores have recently been proposed to predict respiratory syncytial virus (RSV) and influenza virus infections with greater accuracy [1, 9-13]. Despite the suspicion in real life practice that the timing of CARV infection after allo-HCT could be related to increased severity and mortality, there is a lack of strong evidence supporting this belief. Although current risk scores include timing of CARV infection relative to timing of allo-HCT, the cut-off time points vary between different studies. Immunosuppression status in allo-HCT has classically been divided into preengraftment (up to neutrophil recovery), early postengraftment (from engraftment until day +100 ), and late postengraftment $($ after day +100$)$. Based on this classification, several studies have analyzed the impact of timing on severity and mortality in different CARV type scenarios, with inconsistent results. Most studies used the aforementioned cut-off time points of 30 days and/or 100 days and/or 6 months and/or 1 year after stem cell infusion for comparison. Accordingly, some studies found that developing a CARV infection within 30 days after transplant was linked to worse outcome $[6,10]$ whereas others did not [14-18]. In addition, studies using day + 100 as a cut-off time could not show significant association with outcome [19-22], while others observed worse outcome for CARV infections occurring before day +180 [11]. Finally, while some studies found that CARVs beyond 1 year after allo-HCT showed favorable outcomes [23], others did not [14, 17, 24]. In summary, most studies found no effect on patient outcomes regarding the timing of CARV infection development, but due to the discordant results, this issue remains unresolved and warrants further research.

Herein, we evaluate the effect of timing of CARV infection on mortality in a consecutive series of allo-HCT recipients with molecularly-documented CARV infections during the first year after stem cell infusion.

\section{Patients and methods}

\section{Study population and respiratory virus survey protocol}

This was a prospective observational longitudinal epidemiologic study of CARV infections in adult ( $>18$ years) allo-HCT recipients conducted at two Spanish transplant centers in Valencia, Spain. The cohort was comprised entirely of consecutive allo-HCT recipients with molecularly-documented CARV infections who were prospectively screened for respiratory viruses at the Hospital Clinic Universitari of Valencia (HCUV) between December 2013 and May 2016, and at the Hospital Universitari i Politècnic La Fe in Valencia (HLF) from June 2016 to December 2018.

The CARV survey methodology has previously been described in detail elsewhere [1, 25]. Briefly, from December 2013 at the HCUV and from May 2016 at the HLF, all consecutive patients with URTD and/or LRTD symptoms were encouraged to undergo detailed respiratory virus screening through molecular testing. We prospectively recorded clinical and biological characteristics at the time of CARV screening, including transplant characteristics, variables included in the immunodeficiency scoring index (ISI) [9], hospital admission, immunosuppressant drugs, and signs or symptoms of acute or chronic GvHD. The local ethics committee approved the study protocol.

\section{Standard of care for infection control practices and management of CARV infections}

In December 2013 at the HCUV and in May 2016 at the HLF, we implemented the medical information/education for recipients and caregivers explaining in detail about the risks of having RVI in the context of immunosuppression. Specific information included a description of respiratory symptoms that merits urgent notification to the transplant team and recommendations concerning screening of respiratory virus, available therapies, and infectious prevention control measures for patients and health caregivers. Appropriate precautions and infection control measures for preventing CARV transmission in allo-HCT recipients with respiratory symptoms were instituted at both centers according to published guidelines [26].

As per protocol, we started oseltamivir in allo-HCT recipients with influenza infection provided the respiratory symptoms have not been resolved at the time of microbiological results. Annual influenza vaccination was also recommended to all patients at both transplant centers after the 3rd month following allo-HCT. For patients with moderate to severe GVHD at the time of vaccination program who had received gammaglobulin, antithymocytic 
globuline (ATG) or rituximab within the 3 months before the flu vaccine period, vaccine administration remained at physician discretion [1]. RSV and human parainfluenza virus (HPIV) were managed according to our interventional protocol [27]. Briefly, oral ribavirin was given at a loading dose (maximum daily dose of $30 \mathrm{mg} / \mathrm{kg}$ ) until resolution of respiratory symptoms. Routine prophylactic intravenous immunoglobulins $(0.4 \mathrm{~g} / \mathrm{kg})$ were given when $\mathrm{IgG}$ serum levels were below $300 \mathrm{mg} / \mathrm{dl}$. Ribavirin therapy was instituted in recipients with LRTD caused by RSV or HPIV, whereas patients with URTD must had 3 or more ISI points and/or 2 or more risk factor according to the ECIL-4 guidelines [28], and/or presenting one or more coinfective virus(es) before starting ribavirin.

\section{Cohort selection}

The current study analyzed all consecutive molecularlyproven CARV infectious episodes occurring during the first year after allo-HCT. The recipients/CARV episodes included were relapse-free of baseline disease before the CARV infection diagnosis. The study was conducted from December 12013 until December 26 2018. CARV related complication and survival status were updated on April 28 2019. We counted CARV episodes as follows: in recipients with more than one CARV infectious episode, we censored the episode at the time point of the following CARV episode diagnosis. For recipients who died after developing more than one CARV episode we counted the closest CARV episode for mortality, while the previous ones were censored as alive at the time of the following CARV episode.

\section{Definitions}

URTD was defined as a combination of upper respiratory symptoms (rhinorrhea, sinusitis, otitis, or pharyngitis) as well as positive CARV diagnosis by PCR test in respiratory samples, and absence of LRTD symptoms and/or any indication of pulmonary infiltrates in chest X-ray or CT scan radiology results. We classified LRTD as possible, probable or confirmed, as previously described [17]. There were no probable episodes because bronchoscopies were not performed in patients without radiological proof of pulmonary involvement. Respiratory virus infection episodes were defined according to ECIL-4 recommendations [28].

\section{Technical and diagnostic considerations}

CARV testing was performed with three RT-PCR multiplex platforms described in detail elsewhere [1, 25]. At the HCUV samples were tested by RT-PCR using the
Luminex xTAG RVP Fast v1 assay (Luminex Molecular Diagnostics, Toronto, ON, Canada), while at HLF the CLART $^{\oplus}$ PneumoVir DNA array assay (Genomica, Coslada, Spain) was performed and interpreted during the study period following the manufacturer's recommendations. The Luminex xTAG RVP Fast v1 assay can detect adenoviruses (ADVs); human bocavirus (HBoV); human coronavirus (CoV) types 229E, HKU1, NL63, and OC43; influenza $\mathrm{A}$ virus $\mathrm{A} / \mathrm{H} 1 \mathrm{~N} 1, \mathrm{~A} / \mathrm{H} 3 \mathrm{~N} 2$, and other influenza viruses (non-subtypificable); influenza $\mathrm{B}$ virus; human metapneumovirus (HMPV) A and B; HPIV 1, 2, 3, and 4A-4B; RSV A-B; and enterovirus/rhinovirus (EvRh). The CLART ${ }^{\oplus}$ PneumoVir DNA array assay differs from the Luminex xTAG RVP Fast assay in that it detects influenza $\mathrm{C}$ virus but does not allow the detection of the alphacoronavirus NL63 virus and the betacoronaviruses HKU1 and OC43. The CLART ${ }^{\oplus}$ PneumoVir is able to discriminate between rhinovirus and enterovirus genus, and it permits the identification of the new influenza A/H1N1v. At HLF from July 2018 onwards CARV screening in allo-HCT recipients was performed by BioFire FilmArray ${ }^{\oplus}$ Respiratory Panel (BioFire Diagnostics (a bioMerieux company), Salt Lake City, UT), which is able to detect 15 respiratory viruses: influenza virus types $\mathrm{A}, \mathrm{B}$, and $\mathrm{C}$ (with influenza A subtyping), $\mathrm{ADV}, \mathrm{HCoV}$ HKU1, NL63, 229E and OC43, HMPV, EvRh, HPIV types 1-4 and RSV, and also detects three bacteria: Mycoplasma pneumoniae, Chlamydia pneumoniae, and Bordetella pertussis.

\section{Endpoints and statistical analysis}

The primary objective of the study was to assess 3-month all-cause mortality from time of CARV diagnosis according to the timing of CARV infection during the first year after allo-HCT. Secondary endpoints included identifying other risk factors for all-cause mortality and overall survival (OS).

Frequencies were compared using the $\chi^{2}$ test (Fisher exact test) for categorical variables. Differences between medians were compared using the Mann-Whitney $U$ test. Univariate and multivariate analyses of how clinical and biological variables associated with 3-month all-cause mortality were calculated using logistic regression models. For multivariate analysis, only variables with parameter estimates showing a $P$ value $\leq 0.10$ in the univariate analysis were finally included. Two-sided exact $P$ values were reported and $P$ values $\leq 0.05$ were considered statistically significant. The probability of OS according to the timing of CARV infection was estimated from the time of CARV detection using Kaplan-Meier curves [29] and univariate comparisons were made with the log-rank test [30]. The data was analyzed with the SPSS (version 20.0) statistical package. 


\section{Results}

Overall, 326 allo-HCT recipients developed 804 episodes of upper and/or lower respiratory symptoms which were screened for CARVs. Among them, 298 allo-HCT recipients (91\%) developed at least one episode of molecularly documented CARV, accounting for $633 \mathrm{CARV}$ infection episodes $(78 \%)$ during the six-year study period. After excluding 36 episodes $(6 \%)$ that had occurred after baseline disease relapse, overall we considered 287 allo-HCT recipients with 597 consecutive molecularly-documented CARV infectious episodes. For study purposes, we excluded 221 CARV episodes occurring after the first year of transplant, including 54 allo-HCT recipients who developed their first CARV infection episode beyond the first year of transplant. Finally, we included 233 allo-HCT recipients who had developed 376 molecularly documented CARV infections during the first year after allo-HCT.

Clinical and transplant characteristics of the series are detailed in Table 1 . The study population comprised a highrisk cohort, since $70 \%$ of the recipients were allografted from alternative donors [adult unrelated donor (URD), single cord blood units, or haplo-identical family donors]. Human leukocyte antigen (HLA)-mismatch between donor and recipient (considering high resolution typing of HLA A, B, C, DRB1, and DQB1) represented $47 \%$ of the cohort. Sixty-one recipients (26\%) received ATG because of URDrecipient HLA mismatch $(n=8)$, cord blood transplant $(n$ $=47$ ), or HLA-identical sibling in the context of a clinical trial $(n=6)$.

\section{CARV respiratory virus infection characteristics}

CARV episodes were diagnosed at a median of 139 days after stem cell infusion (range, day -7 to +353 ). Twohundred and thirty-six CARV episodes $(63 \%)$ were limited to the URTD whereas 140 (37\%) had LRTD involvement (72 possible LRTD and 68 proven LRTD). Table 2 summarizes the most common CARV types isolated, and the corresponding rates of URTD and LRTD.

\section{CARV infection episode characteristics and 3-month all-cause mortality according to time of transplantation}

In order to identify the most critical period for CARV infection severity we analyzed characteristics and mortality rate according to the time of diagnosis for each one during the first 12 months of transplant. As shown in Fig. 1, most CARV episodes occurred within the first 6 months (227 out of $376,60 \%$ ), particularly in the 1 st month after transplantation (63 out of $376,28 \%$ ). CARV LRTD was also more commonly observed within the first
Table 1 Patient characteristics

\begin{tabular}{|c|c|}
\hline Characteristics & $(n=233)$ \\
\hline Age (years), median (range) & $47(18-70)$ \\
\hline Male, $n(\%)$ & $130(56)$ \\
\hline \multicolumn{2}{|l|}{ Baseline disease, $n(\%)$} \\
\hline AL/MDS/MPD & $123(53) / 16(7) / 15(6)$ \\
\hline Lymphoid disorders & $75(32)$ \\
\hline Others & $4(2)$ \\
\hline \multicolumn{2}{|l|}{ Disease status at transplant, $n(\%)$} \\
\hline $\mathrm{CR}$ & $161(69)$ \\
\hline PR & $32(14)$ \\
\hline Refractory/active disease & $40(17)$ \\
\hline Prior ASCT, $n(\%)$ & $57(25)$ \\
\hline \multicolumn{2}{|l|}{ Period of transplant, $n(\%)$} \\
\hline 2017-2018 & $86(37)$ \\
\hline 2015-2016 & $62(27)$ \\
\hline 2013-2014 & $63(27)$ \\
\hline 2012 & $22(9)$ \\
\hline \multicolumn{2}{|l|}{ Conditioning regimen, $n(\%)$} \\
\hline RIC & $105(45)$ \\
\hline \multicolumn{2}{|l|}{ Type of donor, $n(\%)$} \\
\hline HLA-identical sibling donor & $70(30)$ \\
\hline Unrelated donor & $59(25)$ \\
\hline Umbilical cord blood & $51(22)$ \\
\hline Haploidentical family donor & $53(23)$ \\
\hline PB stem cell source, $n$ (\% & $175(75)$ \\
\hline HLA fully-matched, $n(\%)$ & $123(53)$ \\
\hline ATG as a part of conditioning regimen, $n(\%)$ & $61(26)$ \\
\hline \multicolumn{2}{|l|}{ GvHD prophylaxis, $n(\%)$} \\
\hline Sir-Tac & $24(10)$ \\
\hline $\mathrm{CsA}+\mathrm{MTX}$ & $43(18)$ \\
\hline Post-Cy & $106(46)$ \\
\hline CsA + PDN and others & $60(26)$ \\
\hline $\begin{array}{l}\text { Number CARVs episodes by days after SC infusion, } \\
n(\%)\end{array}$ & 376 \\
\hline Day -7 until Day +30 & $63(17)$ \\
\hline Day +31 to Day +60 & $28(7)$ \\
\hline Day +61 to Day +90 & $39(10)$ \\
\hline Day +91 to Day +180 & $96(26)$ \\
\hline Day +181 to Day +356 & $150(40)$ \\
\hline Number of LRTD CARV episodes & 140 \\
\hline $\begin{array}{l}\text { Median time from allo-HSCT to CARV, days } \\
\text { (range) }\end{array}$ & $139(-7$ to 353$)$ \\
\hline Median F/U after CARV, days (range) & $275(0-2356)$ \\
\hline
\end{tabular}

$A L$ acute leukemia, $M D S$ myelodysplastic syndrome, $M P D$ myeloproliferative disease, $C R$ complete remission, $P R$ partial remission, $A S C T$ autologous stem cell transplantation, $R I C$ reduced intensity conditioning, ATG anti-thymocyte globuline, Sir sirolimus, Tac tacrolimus, $C s A$ cyclosporine A, MTX methotrexate, Post-Cy posttransplant cyclophosphamide, PDN prednisone, SC stem cell, allo-HSCT allogeneic hematopoietic stem cell transplantation, $C A R V$ community-acquired respiratory virus, $F / U$ follow-up, $I F D$ invasive pulmonary infectious fungal disease, $L R T D$ lower respiratory tract disease

6 months (83 out of $140,59 \%)$. We likewise perceived that the 3-month all-cause mortality rate was clustered mainly in the first 6 months after transplant in the current series ( 25 out of 30 deaths, $83 \%$ ). This was also observed 
Table 2 Type of CARV and mortality by CARV type and timing of respiratory virus infection and CARV upper or lower respiratory tract disease

\begin{tabular}{|c|c|c|c|c|c|c|c|c|}
\hline & EvRh & RSV & Influ & HPiV & hMPV & $\mathrm{AdV}$ & $\mathrm{HCoV}$ & $\mathrm{HBoV}$ \\
\hline Number of episodes, $n(\%)^{\mathrm{a}}$ & $145(39)$ & $100(27)$ & $58(15)$ & $65(17)$ & $38(10)$ & $8(2)$ & $24(6)$ & $10(3)$ \\
\hline 90-day overall mortality, $n(\%)^{\mathrm{a}}$ & $10(7)$ & $12(12)$ & $3(5)$ & $5(8)$ & $3(8)$ & $1(13)$ & $2(8)$ & 0 \\
\hline CARV URTD, $n(\%)^{\mathrm{a}}$ & $102(70)$ & $54(54)$ & $38(65)$ & $37(72)$ & $21(55)$ & $2(25)$ & $18(75)$ & $9(90)$ \\
\hline 90-day overall mortality, $n(\%)^{\mathrm{a}}$ & $3(3)$ & $2(4)$ & $1(3)$ & $2(5)$ & $1(5)$ & 0 & $1(6)$ & 0 \\
\hline CARV URTD from day -7 until day $+180, n^{\mathrm{a}}$ & $64(63)$ & $30(56)$ & $18(47)$ & $22(59)$ & $14(67)$ & $1(50)$ & $10(56)$ & $5(56)$ \\
\hline 90-day overall mortality, $n^{\text {a }}$ & $3(5)$ & $2(7)$ & $1(5)$ & $1(4)$ & $1(7)$ & 0 & $1(10)$ & 0 \\
\hline CARV URTD from day +181 until 1 year, $n^{\mathrm{a}}$ & $38(37)$ & $24(44)$ & $20(53)$ & $15(41)$ & $7(33)$ & $1(50)$ & $8(44)$ & $4(44)$ \\
\hline 90-day overall mortality, $n(\%)^{\mathrm{a}}$ & 0 & 0 & 0 & $1(7)$ & 0 & 0 & 0 & 0 \\
\hline CARV LRTD, $n(\%)^{\mathrm{a}}$ & $43(30)$ & $46(46)$ & $20(35)$ & $18(28)$ & $17(45)$ & $6(75)$ & $6(25)$ & $1(10)$ \\
\hline 90-day overall mortality, $n(\%)^{\mathrm{a}}$ & $7(16)$ & $10(22)$ & $2(10)$ & $3(17)$ & $2(12)$ & $1(17)$ & $1(17)$ & 0 \\
\hline CARV LRTD from day -7 until day $+180, n^{\mathrm{a}}$ & $29(67)$ & $29(63)$ & $14(70)$ & $13(72)$ & $13(76)$ & $4(67)$ & $3(50)$ & $1(100)$ \\
\hline 90-day overall mortality, $n(\%)^{\mathrm{a}}$ & $5(17)$ & $9(31)$ & $2(14)$ & $2(15)$ & $2(15)$ & $1(25)$ & $1(33)$ & 0 \\
\hline CARV LRTD from day +181 until 1 year, $n^{\text {a }}$ & $14(33)$ & $17(37)$ & $6(30)$ & $5(28)$ & $4(24)$ & $2(33)$ & $3(50)$ & 0 \\
\hline 90-day overall mortality, $n(\%)^{\mathrm{a}}$ & $2(14)$ & $1(6)$ & 0 & $1(20)$ & 0 & 0 & 0 & 0 \\
\hline
\end{tabular}

$C A R V$ community-acquired respiratory virus, $E v R h$ enterovirus/rhinovirus, $A D V$ adenovirus, $R S V$ respiratory syncytial virus, $H P i V$ human parainfluenza virus, $h M P V$ human metapneumovirus, $H C o V$ human coronavirus, Influ human influenza virus, $A d V$ adenovirus, IFD invasive pulmonary fungal disease, URTD upper respiratory tract disease, $L R T D$ lower respiratory tract disease

${ }^{a}$ The sum total of the episodes does not match the overall number of episodes $(n=376)$ since multiple CARVs were detected in the same respiratory sample in 72 (19\%) CARV episodes. 90-day all-cause mortality after CARV coviral infection was $8 \%$ (6 out of 72). Forty-one covirus infectious episodes occurred within the first 6 months after stem cell infusion and mortality was 15\% (6 out of 41). Two (5\%) out of 44 with URTD and four (14\%) out of 28 patients with LRTD CARV coviral infection died. Finally, 4 (24\%) out of 17 patients with LRTD CARV coviral infection occurring within the first 6 months after stem cell infusion died

Fig. 1 Characteristics of CARV infection episodes and mortality CARV detection after alloHSCT. URTD upper respiratory tract disease, LRTD lower respiratory tract disease rate according to month of

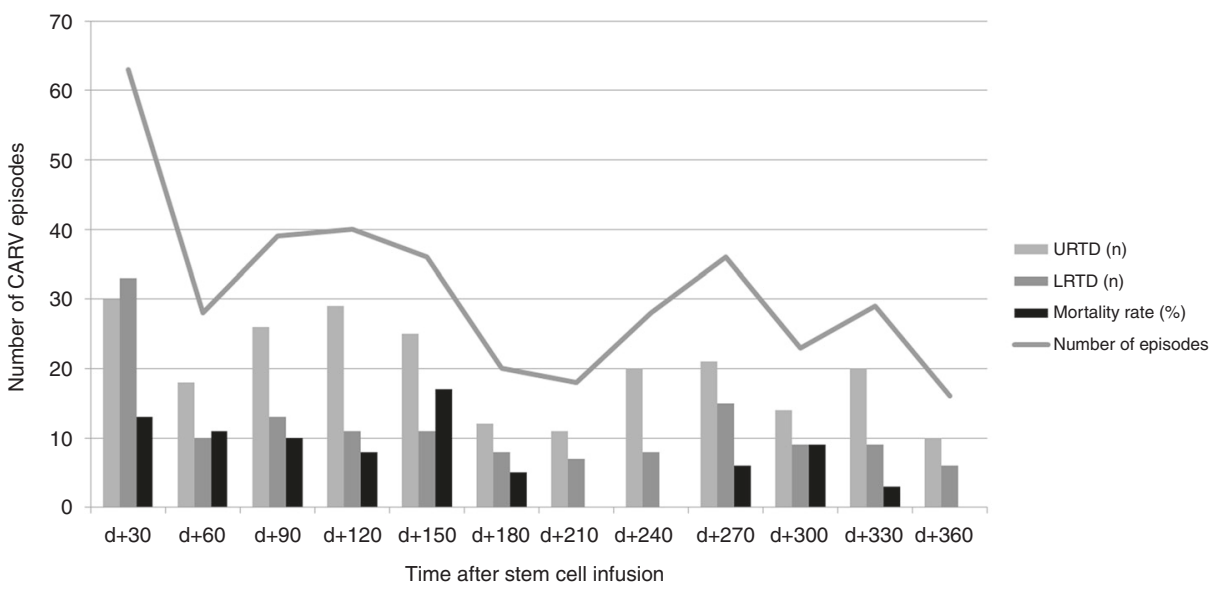

when we analyzed different CARV types without statistically significant differences in mortality (see Table 2). In fact, 8 out of 10 deaths with detected EvRh developed the respiratory virus infection within the first 6 months of transplant, as was also the case in 11 of 12 deaths with RSV, in all three cases with Influ, three out of five with PIV, all three cases with hMPV, both cases with $\mathrm{HCoV}$, and in the only with ADV case. In light of these observations, we choose 6 months as the optimal cut-off time for comparison purposes.

\section{Characteristics of CARV infection episodes according to the 6-month cut-off point}

Patients' clinical and biological characteristics according to whether CARV infections developed earlier or later than 6 months after transplant are summarized in Table 3. As expected, the group who developed CARV infections in the first 6 months had significantly higher incidence of severe immunosuppression-related factors. Use of HLAmismatched donors and ISI score variables (lymphopenia, 
Table 3 Clinical and biological characteristics of respiratory virus infection episodes in allo-HSCT recipients according to timing of CARV

\begin{tabular}{|c|c|c|c|}
\hline & $\begin{array}{l}\text { CARV before } \\
\mathrm{d}+180 \\
(n=227)\end{array}$ & $\begin{array}{l}\text { CARV after } \\
\mathrm{d}+180 \\
(n=149)\end{array}$ & $P$ value \\
\hline \multicolumn{4}{|l|}{ Transplant characteristics } \\
\hline ATG as part of conditioning & $56(25)$ & $34(23)$ & 0.4 \\
\hline GVHD prophylaxis & & & 0.06 \\
\hline Sir-Tac & $15(7)$ & $22(15)$ & \\
\hline $\mathrm{CsA}+\mathrm{MTX}$ & $32(14)$ & $24(16)$ & \\
\hline Post-Cy & $121(53)$ & $71(48)$ & \\
\hline CsA + PDN and others & $59(26)$ & $32(22)$ & \\
\hline HLA mismatch, $n(\%)$ & $116(51)$ & $65(44)$ & 0.09 \\
\hline Type of donor, $n(\%)$ & & & 0.3 \\
\hline HLA-identical sibling donor & $64(28)$ & $51(34)$ & \\
\hline Unrelated donor & $50(22)$ & $40(27)$ & \\
\hline Umbilical cord blood & $50(22)$ & $26(17)$ & \\
\hline Haplo-identical family donor & $63(28)$ & $32(21)$ & \\
\hline \multicolumn{4}{|l|}{$\begin{array}{l}\text { Immunodeficiency scoring } \\
\text { index, } n(\%)^{\mathrm{a}}\end{array}$} \\
\hline $\mathrm{ANC}<0.5 \times 10^{9} / \mathrm{L}(3 \mathrm{pts})$ & $46(20)$ & $5(3)$ & 0.001 \\
\hline $\mathrm{ALC}<0.2 \times 10^{9} / \mathrm{L}(3 \mathrm{pts})$ & $68(30)$ & $12(8)$ & 0.001 \\
\hline Age $\geq 40$ year $(2 \mathrm{pts})$ & $150(66)$ & $101(68)$ & 0.4 \\
\hline $\begin{array}{l}\text { Myeloablative conditioning } \\
\text { regimen }(1 \mathrm{pt})\end{array}$ & $133(59)$ & $76(51)$ & 0.09 \\
\hline GvHD (acute or chronic; 1 pt) & $82(36)$ & $87(58)$ & 0.001 \\
\hline Corticosteroids (1 pt) & $86(38)$ & $51(34)$ & 0.3 \\
\hline $\begin{array}{l}\text { Recent or preengraftment allo- } \\
\text { HSCT ( } 1 \text { pt) }\end{array}$ & $64(28)$ & 0 & 0.001 \\
\hline ISI, $n(\%)$ & & & 0.001 \\
\hline Low risk (0-2) & $78(34)$ & $57(38)$ & \\
\hline Moderate risk (3-6) & $96(42)$ & $83(56)$ & \\
\hline High risk $(7-12)$ & $53(23)$ & $9(6)$ & \\
\hline \multicolumn{4}{|l|}{ Other characteristics ${ }^{\mathrm{a}}$} \\
\hline On IS, $n(\%)$ & $208(92)$ & $104(70)$ & 0.001 \\
\hline $\mathrm{ALC}<0.1 \times 10^{9} / \mathrm{L}, n(\%)$ & $51(22)$ & $4(3)$ & 0.001 \\
\hline $\mathrm{ALC}<0.5 \times 10^{9} / \mathrm{L}, n(\%)$ & $119(52)$ & $25(17)$ & 0.001 \\
\hline \multicolumn{4}{|l|}{$\begin{array}{l}R V I \text { characteristics and clinical } \\
\text { consequences }\end{array}$} \\
\hline CARV LRTD, $n(\%)$ & $87(38)$ & $53(36)$ & 0.3 \\
\hline Possible & $40(46)$ & $32(60)$ & \\
\hline Proven & $47(54)$ & $21(40)$ & \\
\hline Hospital admission, $n(\%)$ & $115(51)$ & $40(27)$ & 0.001 \\
\hline Fever during CARV, $n(\%)$ & $148(65)$ & $82(55)$ & 0.03 \\
\hline $\begin{array}{l}\text { Day }+90 \text { overall mortality rate, } \\
n(\%)\end{array}$ & $25(11)$ & $5(3)$ & 0.005 \\
\hline
\end{tabular}

$C A R V$ community-acquired respiratory virus, IFD invasive pulmonary infectious fungal disease, $A T G$ antithymocyte globuline, $G v H D$ graftversus-host disease, Sir sirolimus, Tac tacrolimus, CsA cyclosporine A, MTX methotrexate, Post-Cy post-transplant cyclophosphamide, $P D N$ prednisone, Allo-HSCT allogeneic hematopoietic stem cell transplantation, $A N C$ absolute neutrophil count, $A L C$ absolute lymphocyte count, $I S$ immunosuppressants, LRTD lower respiratory tract disease

${ }^{\mathrm{a}}$ All variables were captured at the time of CARV diagnosis

active GVHD, steroid therapy) were significantly overrepresented in the early CARV infection group ( $p \leq 0.05$ for all comparisons). CARV infection episodes in this group were more frequently accompanied by fever and/or hospitalization $(p \leq 0.03)$.

\section{Analysis of risk factors for 3-month all-cause mortality}

Logistic regression univariate and multivariate analyses of risk factors for 3-month all-cause mortality after CARV infection episodes and LRTD are shown in Table 4.

We studied the 376 evaluable recipient/episode pairs to identify transplant and CARV conditions associated with mortality. Multivariate analysis identified four RFs: use of ATG as a part of conditioning regimen [OR 2.8, 95\% C.I. $1.21-6.4, p=0.01]$, CARV lower respiratory tract disease (OR 3.4, 95\% C.I. 1.4-8.4, $p=0.007$ ), CARV infection during the first 6 months of transplant (OR 3.04, 95\% C.I. $1.1-8.7, p=0.03)$, and absolute lymphocyte count $<0.2 \times$ $10^{9} / \mathrm{L}$ (OR 2.4, 95\% C.I. $1-5.3, p=0.04$ ).

We analyzed episodes of CARV involving LRTD to determine risk factors for mortality in these cases $(n=$ 140). Multivariate analysis identified three independent factors associated with mortality. Again, ATG and early CARV infection (within the first 6 months) were associated with higher mortality (OR 3.6, 95\% C.I. 1.2-10.6, $p=$ 0.019 and OR 2.7, 95\% C.I. 1-15.1, $p=0.05$, respectively). Finally, high-risk ISI category was also associated with higher probability of mortality (OR 4, 95\% C.I. $1.3-10.6, p=0.017$ ).

Developing CARV within the first 6 months of transplantation had a negative effect on OS at 3 months after CARV diagnosis. OS was $88 \%$ for early CARV infection episodes vs. $96 \%$ for those with late CARV infection $(p=$ 0.009). OS was significantly lower in allo-HCT recipients with early CARV LRTD than in those with late CARV LRTD (78\% vs. 92\%, respectively, $p=0.04$ ) (Fig. 2a, b).

\section{Timing of mortality and cause of death}

Fifteen recipients died by day 30 after CARV diagnosis. Six additional recipients died at day 60 whereas nine more recipients died by day 90 after CARV diagnosis. We did not observed significant differences among the cause of death according to the time of death after CARV detection. Eight and 22 recipients with URTD or LRTD died, respectively. Three, one and five and twelve, five and five recipients with URTD or LRTD died by day 30,60 , and 90 , respectively. However, median time from CARV detection to death among recipients with URTD or LRTD was not statistically different (45 days vs. 37 days, $p=0.4$, respectively).

Cause of death in recipients who die by day 30 were respiratory failure due to mono or coinfections $(n=10)$, including viral, bacterial and/or fungal coinfections, 
Table 4 Univariate and multivariate analysis of risk factors for mortality after overall CARV infection and LRTD

\begin{tabular}{|c|c|c|c|c|c|c|c|c|}
\hline \multirow[t]{3}{*}{ Variables } & \multicolumn{4}{|c|}{ Log. regr. overall mortality $(n=376)$} & \multicolumn{4}{|c|}{$\begin{array}{l}\text { Log. regr. overall mortality in recipients with LRTD } \\
\text { CARV }(n=140)\end{array}$} \\
\hline & \multicolumn{2}{|c|}{ Univariate analysis } & \multicolumn{2}{|c|}{ Multivariate analysis } & \multicolumn{2}{|c|}{ Univariate analysis } & \multicolumn{2}{|c|}{ Multivariate analysis } \\
\hline & OR (95\% C.I.). & $P$ value & OR (95\% C.I.) & $P$ value & OR (95\% C.I.). & $P$ value & OR (95\% C.I.) & $P$ value \\
\hline \multicolumn{9}{|l|}{ Type of donor, $n(\%)$} \\
\hline HLA-identical sibling donor & 1 & & & & 1 & & & \\
\hline Alternative donor & $6.8(1.58-29)$ & 0.01 & ns & & $8.1(1.1-63)$ & 0.044 & ns & \\
\hline HLA mismatch & $4.8(1.9-12.1)$ & 0.001 & ns & & $5.5(1.5-19.6)$ & 0.008 & ns & \\
\hline ATG as a part of conditioning & $4.9(2.3-10.5)$ & $<0.0001$ & $2.8(1.21-6.4)$ & 0.01 & $4.1(1.6-10.8)$ & 0.003 & $3.6(1.2-10.6)$ & 0.019 \\
\hline GVHD prophylaxis & & & ns & & & & & \\
\hline Sir-Tac & 1 & & & & 1 & & & \\
\hline CsA + MTX & $6.7(0.7-62)$ & 0.1 & & & $3.7(0.5-62)$ & 0.3 & & \\
\hline Post-Cy & $3.2(0.8-11.6)$ & 0.08 & & & $2.2(0.7-13.6)$ & 0.09 & ns & \\
\hline CsA + PDN and others & $0.5(0.15-1.6)$ & 0.2 & & & $0.8(0.45-2.6)$ & 0.2 & & \\
\hline CARV LRTD & $5.3(2.3-12.3)$ & $<0.0001$ & $3.4(1.4-8.4)$ & 0.007 & & & NT & \\
\hline No & 1 & & & & & & & \\
\hline Proven & $4.6(1.7-12.1)$ & 0.002 & NT & & & & & \\
\hline Possible & $6.1(2.3-15.6)$ & 0.001 & NT & & & & & \\
\hline On IS & $3.1(0.7-13.1)$ & 0.13 & & & $1.4(0.3-6.7)$ & 0.6 & & \\
\hline $\mathrm{ALC}<0.5 \times 109 / \mathrm{L}, n(\%)$ & $3.6(1.6-7.8)$ & 0.002 & NT & & $2.4(0.9-6.6)$ & 0.08 & ns & \\
\hline $\mathrm{ALC}<0.2 \times 109 / \mathrm{L}^{\mathrm{a}}$ & $5(2.33-10.8)$ & $<0.0001$ & $2.4(1.03-5.6)$ & 0.04 & $2.9(1.2-7.4)$ & 0.024 & NT & \\
\hline $\mathrm{ALC}<0.1 \times 109 / \mathrm{L}$ & $2.3(0.97-5.5)$ & 0.058 & NT & & $1.2(0.4-3.3)$ & 0.7 & NT & \\
\hline $\mathrm{ANC}<0.5 \times 109 / \mathrm{L}^{\mathrm{a}}$ & $2.6(1.07-6.1)$ & 0.034 & ns & & $2.3(0.8-6.3)$ & 0.11 & NT & \\
\hline Age $\geq 40$ years $^{\mathrm{a}}$ & $0.99(0.4-2.2)$ & 0.9 & & & $1.4(0.5-4.2)$ & 0.5 & & \\
\hline Active GvHD at time of $\mathrm{RVI}^{\mathrm{a}}$ & $1.4(0.7-3)$ & 0.3 & & & $1.3(0.5-3.1)$ & 0.6 & & \\
\hline Periengraftment ${ }^{\mathrm{a}}$ & $2.3(0.98-5.21)$ & 0.054 & ns & & $1.5(.6-4.2)$ & 0.4 & & \\
\hline Allo-HSCT $\leq 6$ months & $3.6(1.33-9.5)$ & 0.01 & $3.04(1.1-8.7)$ & 0.03 & $3.2(1.1-10.3)$ & 0.046 & $2.7(1-15.1)$ & 0.05 \\
\hline Myeloablative $^{\mathrm{a}}$ & $3.8(1.6-8.9)$ & 0.002 & & & $2.7(1.03-7.3)$ & 0.042 & NT & \\
\hline Corticosteroids $^{\mathrm{a}}$ & $3.33(1.5-7.2)$ & 0.002 & & & $3.9(1.3-11.2)$ & 0.012 & NT & \\
\hline Antiviral therapy & $0.6(0.3-1.3)$ & 0.17 & & & $1.16(0.46-2.9)$ & 0.7 & & \\
\hline \multicolumn{9}{|l|}{ Type of RVI } \\
\hline Mono infection & 1 & & & & 1 & & & \\
\hline Coinfection & $1.1(0.4-2.7)$ & 0.9 & & & $0.9(0.3-2.8)$ & 0.8 & & \\
\hline ISI & & & NT & & & & & \\
\hline Low risk $(0-2)$ & 1 & & & & & & & \\
\hline Moderate risk (3-6) & $6.24(2.1-18.6)$ & 0.001 & & & $4(1.02-15.7)$ & 0.047 & $2.8(0.6-12)$ & 0.16 \\
\hline High risk (7-12) & $3.1(1.3-7.1)$ & 0.009 & & & $3.5(1.3-9.9)$ & 0.016 & $4(1.3-10.6)$ & 0.017 \\
\hline
\end{tabular}

C.I. confidence interval, Log. Regr logistic regression model, OR odds ratio, IFD invasive pulmonary fungal disease, $A T G$ antithymocyte globuline, Sir sirolimus, Tac tacrolimus, CsA cyclosporine A, MTX methotrexate, Post-Cy posttransplant cyclophosphamide, PDN prednisone, CARV LRTD community-acquired respiratory virus lower respiratory tract disease, GvHD graft-versus-host disease, Allo-HSCT allogeneic hematopoietic stem cell transplantation, ISI immunodeficiency score index, $A N C$ absolute neutrophil count, $A L C$ absolute lymphocyte count, $n s$ not significant, $N T$ not tested

${ }^{a}$ These variables were included in the ISI score. When ISI score was not significant in the multivariate model; those with $p$ value $<0.1$ in the univariate testing were finally included in the final multivariate model

steroids-refractory GvHD ( $n=3)$, posttransplant lymphoproliferative disease $(n=1)$ and baseline disease progression $(n=1)$. The additional six deaths occurring by day 60 , were due to respiratory failure in the context of pulmonary mono or coinfections $(n=3)$, steroids-refractory GvHD $(n=2)$ and disease relapse $(n=1)$. For the remaining nine patients died by day 90 after CARV detection the causes of death were infectious respiratory failure $(n=4)$, steroidsrefractory $\operatorname{GvHD}(n=2)$, disease relapse $(n=2)$, and pulmonary malignancy $(n=1)$. 

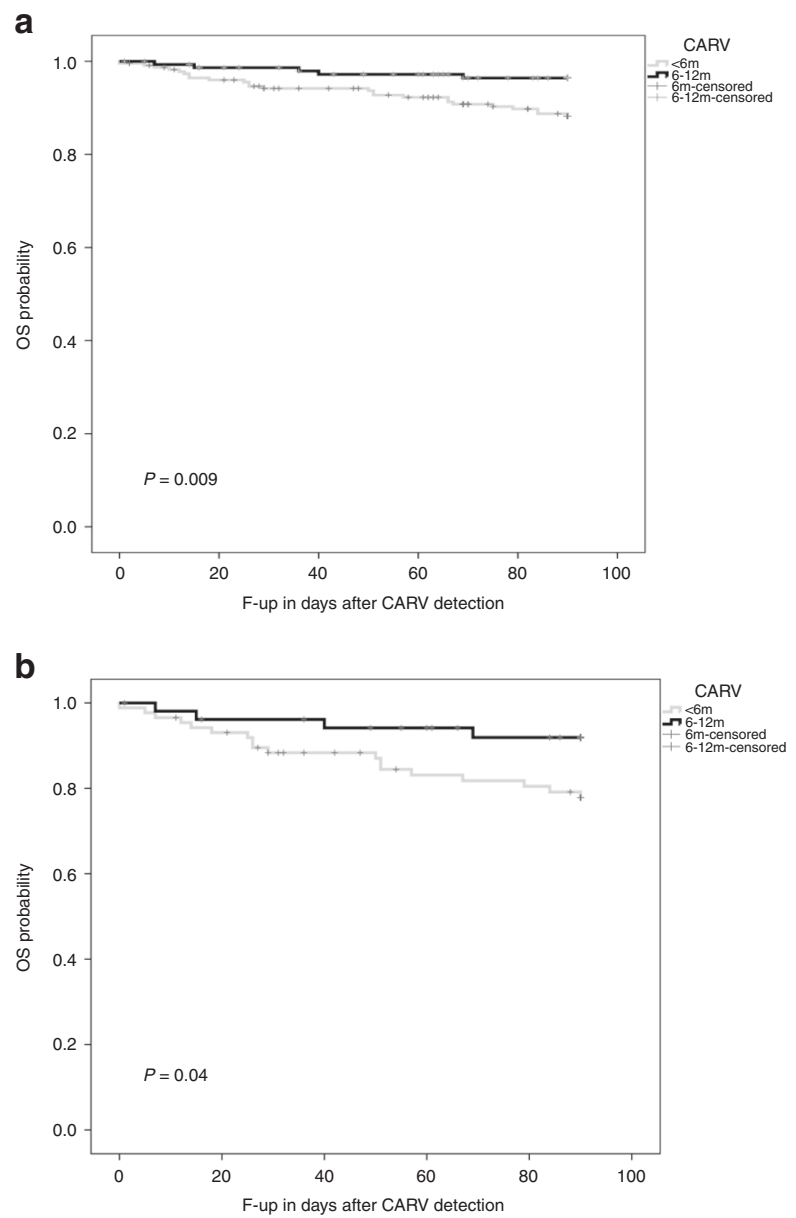

Fig. 2 Overall survival according to development of a community acquired respiratory virus (CARV) respiratory virus infection (RVI) earlier or later than 6 months after transplant and b CARV lower respiratory tract disease (LRTD) earlier or later than 6 months after transplant

\section{Discussion}

This study shows that the timing of CARV infection during the first year of allo-HCT could be one of the major determinants of mortality, irrespective of CARV type. Our multivariate model showed that all-cause mortality at 3 months after CARV detection was significantly higher in recipients who developed CARV infection within the first 6 months of transplant. In addition, we found that ATG use as part of conditioning regimen, LRTD, and lymphopenia $\left(<0.2 \times 10^{9} / \mathrm{L}\right)$ were also associated with higher risk of mortality. Risk factors for mortality among the CARV LRTD episodes included early CARV LRTD (within the first 6 months), ATG as a part of conditioning regimen, and high-risk ISI category.

Several studies have looked at the effect on mortality of when CARV infection occurs after allo-HCT using heterogeneous cut-off time points, showing discrepant results
$[6,10,11,14-24]$. In fact, most of them were unable to find any association between timing and mortality [14-22, 24]. In contrast, our detailed analysis of 3-month all-cause mortality conducted monthly during the first year revealed that most deaths occurred in recipients who developed a CARV infection within the first 6 months of transplant (see Fig. 1). We confirmed this observation in our multivariate model by including the " 6 months" variable as an optimal cut-off time point in both overall and CARV LRTD. The differences observed between our study and others could be explained by two issues, the first being the risk of selection bias inherent to retrospective studies, with the inclusion of severe cases at any time after transplant. The power to detect differences in mortality according to the timing of CARV infection in severe cases is likely to be limited. Second, the most common cut-off time points used for comparison in prior reports were "day 0 to +30 vs. day +31 to +365 vs. $>$ day +365 " and before or after day +100 of transplant. The selected intervals of both comparators overlap with our " 6 month" cut-off. This may have distorted the effect of timing on mortality in prior studies. However, our findings suggest that the aforementioned cut-off time points are probably not optimal enough to differentiate the mortality risk. In fact, we explored these cut-off time points in our cohort, observing no statistical differences in terms of mortality. To our best knowledge, only one study, focused on paramyxovirus CARV infections, included a cut-off of 6 months in their analysis of mortality and found statistically significant differences [11]. This is a predictable result since the 6-month cut-off corresponds to the anticipated length of immunosuppression in most alloHCT recipients.

Given that the inclusion of several CARV types in the current study could be argued to confound our findings, we also independently analyzed the effect of timing in each of the CARVs across our entire cohort. We observed that the timing reported was consistent across all CARV types except HBoV (see Table 2). Our pooled analysis with different CARVs can be justified by the fact that several prior studies comparing mortality and/or OS according to CARV type showed no significant differences [23, 24, 31, 32]. Our findings underscore the need for further prospective studies analyzing in detail the effect of timing of CARV infections on mortality in allo-HCT recipients; this elucidation could be of utmost importance for diagnostic purposes, infectious control measures, and to close monitoring of recipients who develop CARV infections within the first 6 months after transplant. In addition, if timing is confirmed as a major predictive factor for CARV severity, this will certainly raise further unanswered questions. Can the well-established clinical and/or biological risk factors and/or immunodeficiency scores reliably predict mortality due to later developing CARV after transplant? To the best of our 
knowledge, no studies have focused on mortality and risk factors related to CARV infections occurring beyond the first year of transplant.

Except for influenza virus and to some extend for RSV, currently there is an urgent need of new effective antiviral drugs against other CARV types. Although many molecules are under development [33], in the meantime the efforts should be focused on having a timely microbiological diagnostic and preventing CARV transmission, in particular during the first 6 months after transplant. Medical education/information emphasizing what symptoms should be reported immediately to the transplant team, urgent clinical evaluation, and risk stratification within $24-48 \mathrm{~h}$ after the start of respiratory symptoms, rapid application of sensitive diagnostic test and prompt initiation of antiviral drugs in treatable CARV, as well as the early identification and treatment of direct and indirect CARV-related complications may be of value. In addition, there is also an urgent need in educating not only patients and healthcare givers about the risk of transmission associated with CARV infection but especially healthcare workers, managers, workplace health and safety and infection control staff, and administrators [34].

Among the other variables associated with higher mortality in the current study, we confirm that LRTD and lymphopenia remain the most important and validated risk factors for mortality after CARV infection in the allo-HCT setting [4-7]. Besides these, use of ATG showed a direct association with mortality in both overall CARV and those limited to the LRTD. This finding is unsurprising, as ATG produces lymphopenia and a significant delay in $\mathrm{T}$ cell immune reconstitution that lasts longer than 1 year after stem cell infusion [35, 36]. Finally, we have corroborated the value of the high-risk ISI category in predicting mortality in LRTD caused by several CARV types. ISI was developed by investigators from the MD Anderson Cancer Center to predict outcome in allo-HCT recipients with RSV [9]. This score demonstrated value in predicting LRTD progression [1, 10] and mortality [10] in the context of influenza respiratory infection and RSV [13]. However, the failure to differentiate risk of mortality between the moderate-risk and low-risk ISI category $[10,13]$ indicates room for improvement. In the meantime, the high-risk ISI category could be useful to assess the severity of several CARV types with LRTD involvement.

We acknowledge certain limitations of this study, such as the inclusion of several CARV types and the use of three different PCR methods differing (minimally) in analytical performance. Nonetheless, our study has strengths that merit consideration, notably the use of molecular testing and the prospective data collection with a CARV survey.

In conclusion, we provide evidence that the timing of CARV infection after allo-HCT could be of major interest due its considerable impact on outcome. Further prospective studies are warranted to confirm this finding.

\section{Compliance with ethical standards}

Conflict of interest JLP received advisory support for preclinical/ clinical research and financial support to attend the Spanish Hematology Society annual meeting 2018 and the EBMT annual meeting 2019, both from Merck and Sharp Dohme pharmaceutics. The other authors declare that they have no conflict of interest.

Publisher's note Springer Nature remains neutral with regard to jurisdictional claims in published maps and institutional affiliations.

\section{References}

1. Piñana JL, Pérez A, Montoro J, Giménez E, Dolores Gómez M, Lorenzo I, et al. Clinical effectiveness of influenza vaccination after allogeneic hematopoietic stem cell transplantation: a crosssectional prospective observational study. Clin Infect Dis. 2018. https://doi.org/10.1093/cid/ciy792.

2. Chien JW, Martin PJ, Gooley TA, Flowers ME, Heckbert SR, Nichols WG, et al. Airflow obstruction after myeloablative allogeneic hematopoietic stem cell transplantation. Am J Respir Crit Care Med. 2003;168:208-14.

3. Erard V, Chien JW, Kim HW, Nichols WG, Flowers ME, Martin PJ, et al. Airflow decline after myeloablative allogeneic hematopoietic cell transplantation: the role of community respiratory viruses. J Infect Dis. 2006;193:1619-25.

4. Waghmare A, Englund JA, Boeckh M. How I treat respiratory viral infections in the setting of intensive chemotherapy or hematopoietic cell transplantation. Blood. 2016;127:2682-92.

5. Kim YJ, Guthrie KA, Waghmare A, Walsh EE, Falsey AR, Kuypers J, et al. Respiratory syncytial virus in hematopoietic cell transplant recipients: factors determining progression to lower respiratory tract disease. J Infect Dis. 2014;209:1195-204.

6. Shah DP, Ghantoji SS, Shah JN, El Taoum KK, Jiang Y, Popat U, et al. Impact of aerosolized ribavirin on mortality in 280 allogeneic haematopoietic stem cell transplant recipients with respiratory syncytial virus infections. J Antimicrob Chemother. 2013;68:1872-80. 4

7. Piñana JL, Gómez MD, Pérez A, Madrid S, Balaguer-Roselló A, Giménez E, et al. Community-acquired respiratory virus lower respiratory tract disease in allogeneic stem cell transplantation recipient: risk factors and mortality from pulmonary virus-bacterial mixed infections. Transpl Infect Dis. 2018;20: e12926.

8. Martino R, Porras RP, Rabella N, Williams JV, Rámila E, Margall $\mathrm{N}$, et al. Prospective study of the incidence, clinical features, and outcome of symptomatic upper and lower respiratory tract infections by respiratory viruses in adult recipients of hematopoietic stem cell transplants for hematologic malignancies. Biol Blood Marrow Transplant. 2005;11:781-96.

9. Shah DP, Ghantoji SS, Ariza-Heredia EJ, Shah JN, El Taoum KK, Shah PK, et al. Immunodeficiency scoring index to predict poor outcomes in hematopoietic cell transplant recipients with RSV infections. Blood. 2014;123:3263-8.

10. Kmeid J, Vanichanan J, Shah DP, El Chaer F, Azzi J, Ariza-Heredia EJ, et al. Outcomes of influenza infections in hematopoietic cell transplant recipients: application of an immunodeficiency scoring index. Biol Blood Marrow Transplant. 2016;22:542-8. 
11. Spahr Y, Tschudin-Sutter S, Baettig V, Compagno F, Tamm M, Halter J, et al. Community-acquired respiratory paramyxovirus infection after allogeneic hematopoietic cell transplantation: a single-center experience. Open Forum Infect Dis. 2018;5:ofy077. https://doi.org/10.1093/ofid/ofy077.

12. Khanna N, Steffen I, Studt JD, Schreiber A, Lehmann T, Weisser $\mathrm{M}$, et al. Outcome of influenza infections in outpatients after allogeneic hematopoietic stem cell transplantation. Transpl Infect Dis. 2009;11:100-5.

13. Vakil E, Sheshadri A, Faiz SA, Shah DP, Zhu Y, Li L, et al. Risk factors for mortality after respiratory syncytial virus lower respiratory tract infection in adults with hematologic malignancies. Transpl Infect Dis. 2018;20:e12994.

14. Ogimi C, Greninger AL, Waghmare AA, Kuypers JM, Shean RC, $\mathrm{Xie} \mathrm{H}$, et al. Prolonged shedding of human coronavirus in hematopoietic cell transplant recipients: risk factors and viral genome evolution. J Infect Dis. 2017;216:203-9.

15. Lehners N, Schnitzler P, Geis S, Puthenparambil J, Benz MA, Alber B, et al. Risk factors and containment of respiratory syncytial virus outbreak in a hematology and transplant unit. Bone Marrow Transplant. 2013;48:1548-53.

16. Ustun C, Slabý J, Shanley RM, Vydra J, Smith AR, Wagner JE, et al. Human parainfluenza virus infection after hematopoietic stem cell transplantation: risk factors, management, mortality, and changes over time. Biol Blood Marrow Transplant. 2012; 18:1580-8.

17. Seo S, Xie H, Campbell AP, Kuypers JM, Leisenring WM, Englund JA, et al. Parainfluenza virus lower respiratory tract disease after hematopoietic cell transplant: viral detection in the lung predicts outcome. Clin Infect Dis. 2014;58:1357-68.

18. Seo S, Campbell AP, Xie H, Chien JW, Leisenring WM, Englund $\mathrm{JA}$, et al. Outcome of respiratory syncytial virus lower respiratory tract disease in hematopoietic cell transplant recipients receiving aerosolized ribavirin: significance of stem cell source and oxygen requirement. Biol Blood Marrow Transplant. 2013;19:589-96. https://doi.org/10.1016/j.bbmt.2012.12.019. Epub 2013 Jan 5

19. Jacobs SE, Soave R, Shore TB, Satlin MJ, Schuetz AN, Magro C, et al. Human rhinovirus infections of the lower respiratory tract in hematopoietic stem cell transplant recipients. Transpl Infect Dis. 2013;15:474-86.

20. Sheshadri A, Chemaly RF, Alousi AM, Shah PK, Rondon G, Bashoura L, et al. Pulmonary Impairment after respiratory viral infections is associated with high mortality in allogeneic hematopoietic cell transplant recipients. Biol Blood Marrow Transplant. 2019;25:800-9.

21. Waghmare A, Xie H, Kuypers J, Sorror ML, Jerome KR, Englund $\mathrm{JA}$, et al. Human rhinovirus infections in hematopoietic cell transplant recipients: risk score for progression to lower respiratory tract infection. Biol Blood Marrow Transplant. 2018. pii: S1083-8791 (18)30804-8.

22. Campbell AP, Guthrie KA, Englund JA, Farney RM, Minerich EL, Kuypers J, et al. Clinical outcomes associated with respiratory virus detection before allogeneic hematopoietic stem cell transplant. Clin Infect Dis. 2015;61:192-202. https://doi.org/10.1093/ cid/civ272.

23. Ogimi C, Waghmare AA, Kuypers JM, Xie H, Yeung CC, Leisenring WM, et al. Clinical significance of human coronavirus in bronchoalveolar lavage samples from hematopoietic cell transplant recipients and patients with hematologic malignancies. Clin Infect Dis. 2017;64:1532-9. https://doi.org/10.1093/cid/cix160.

24. Seo S, Waghmare A, Scott EM, Xie H, Kuypers JM, Hackman $\mathrm{RC}$, et al. Human rhinovirus detection in the lower respiratory tract of hematopoietic cell transplant recipients: association with mortality. Haematologica 2017;102:1120-30. https://doi.org/10. 3324/haematol.2016.153767. Epub 2017 Feb 9

25. Piñana JL, Madrid S, Pérez A, Hernández-Boluda JC, Giménez E, Terol MJ, et al. Epidemiologic and clinical characteristics of coronavirus and bocavirus respiratory infections after allogeneic stem cell transplantation: a prospective single-center study. Biol Blood Marrow Transplant. 2018;24:563-70.

26. Tomblyn M, Chiller T, Einsele H, Gress R, Sepkowitz K, Storek J, et al. Center for International Blood and Marrow Research; National Marrow Donor program; European Blood and MarrowTransplant Group; American Society of Blood and Marrow Transplantation; Canadian Blood and Marrow Transplant Group; Infectious Diseases Society of America; Society for Healthcare Epidemiology of America; Association of Medical Microbiology and Infectious Disease Canada; Centers for Disease Control and Prevention. Guidelines for preventing infectious complications among hematopoietic cell transplant recipients: a global perspective. Biol Blood Marrow Transplant. 2009;15:1143-238.

27. Piñana JL, Hernández-Boluda JC, Calabuig M, Ballester I, Marín M, Madrid S, et al. A risk-adapted approach to treating respiratory syncytial virus and human parainfluenza virus in allogeneic stem cell transplantation recipients with oral ribavirin therapy: a pilot study. Transpl Infect Dis. 2017;19:e12729.

28. Hirsch $\mathrm{HH}$, Martino R, Ward $\mathrm{KN}$, Boeckh M, Einsele $\mathrm{H}$, Ljungman P. Fourth European Conference on infections in leukaemia (ECIL-4): guidelines for diagnosis and treatment of human respiratory syncytial virus, parainfluenza virus, metapneumovirus, rhinovirus, and coronavirus. Clin Infect Dis. 2013;56:258-66.

29. Klein JP, Rizzo JD, Zhang MJ, Keiding N. Statistical methods for the analysis and presentation of the results of bone marrow transplants. Part I: unadjusted analysis. Bone Marrow Transpl. 2001;28:909-15.

30. Klein JP, Rizzo JD, Zhang MJ, Keiding N. Statistical methods for the analysis and presentation of the results of bone marrow transplants. Part 2: regression modeling. Bone Marrow Transpl. 2001;28:1001-11.

31. Williams JV, Martino R, Rabella N, Otegui M, Parody R, Heck $\mathrm{JM}$, et al. A prospective study comparing human metapneumovirus with other respiratory viruses in adults with hematologic malignancies and respiratory tract infections. $J$ Infect Dis. 2005;192:1061-5.

32. Renaud C, Xie H, Seo S, Kuypers J, Cent A, Corey L, et al. Mortality rates of human metapneumovirus and respiratory syncytial virus lower respiratory tract infections in hematopoietic cell transplantation recipients. Biol Blood Marrow Transplant. 2013; 19:1220-6.

33. Brendish NJ1, Clark TW. Antiviral treatment of severe non-influenza respiratory virus infection. Curr Opin Infect Dis. 2017;30:573-8.

34. Jiang L, McGeer A, McNeil S, Katz K, Loeb M, Muller MP, et al. Which healthcare workers work with acute respiratory illness? Evidence from Canadian acute-care hospitals during 4 influenza seasons: 2010-2011 to 2013-2014. Infect Control Hosp Epidemiol. 2019;18:1-8. https://doi.org/10.1017/ice.2019.141. Canadian Healthcare Worker Study Group.

35. Thomson BG, Robertson KA, Gowan D, Heilman D, Broxmeyer $\mathrm{HE}$, Emanuel D, et al. Analysis of engraftment, graft-versus-host disease, and immune recovery following unrelated donor cord blood transplantation. Blood. 2000;96:2703-11.

36. Mohty M, Bay JO, Faucher C, Choufi B, Bilger K, Tournilhac O, et al. Graft-versus-host disease following allogeneic transplantation from HLAidentical sibling with antithymocyte globulin-based reduced-intensity preparative regimen. Blood. 2003;102:470-6. 